\title{
STUDY OF ECTOPARASITIC FAUNA DIVERSITY IN WILD BIRDS FROM VARIOUS ANTHROPIC BIOTOPES OF THE REPUBLIC OF MOLDOVA
}

\author{
Ştefan Rusu, Dumitru Erhan, Maria Zamornea, Elena Gherasim, Viorelia Rusu
}

Institute of Zoology, Chişinău, Republic of Moldova, e-mail: rusus1974@yahoo.com

\begin{abstract}
The results of the complex parasitological studies conducted in the wild birds of hunting interest as well as domestic birds demonstrates that the phenomenon of the poliparasitism is a static one, although the poliparasitic quantitative and qualitative structure is continuously changing. This is due to instantaneous contact of the wild birds with the domestic ones, irregular deparasiting of domestic animals and their crates, reduced areas for animals breading and maintenance that ensures the permanent contact with the wild birds as the infestation source.

The findings of the study on the diversity of the ectoparasitic fauna in wild birds from various anthropic biotopes of Moldova revealed that the wild and domestic birds are infested with multiple parasites and that the most identified parasitoses are common for major birds of hunting interest as well as for the domestic birds.
\end{abstract}

\section{Introduction}

The parasitoses represent the most frequent diseases in wild and domestic birds that cause essential economic losses [8]. Some authors indicate that among the birds, gallinaceans are especially sensible to infestation with ectoparasites, compared to palmipeds [1, 3-8].

The effective evaluation of animal population species of the hunting interest implies awareness about behavior, relationships among the species and components of the ecosystems they belong to. The parasitic fauna of wild birds can influence the birds' population dynamics especially in the long term timeframe [10-13, 19].

The gamasid mites and some species of mallophaga parasiting on domestic and wild birds, serve also the role of vectors of some viral and bacterian pathogens. Therefore it is imperious to identify the mixt invasions with ectoparasites in wild birds continuously spread off and representing one of the actual problems of parasitology and contemporary ecology [9, 14-18, 20].

\section{Materials and methods}

The parasitologic research conducted during years 2015-2019 on ectoparasitic fauna in 257 fancy poultry of hunting interest hold in captivity in various zoological collections in Moldova allowed to track the diversity of their ectoparasitic species.

The ectoparasites were collected from the live birds in line with the new, more informative protocol. The collection and further qualitative research of the ectoparasites in various stages of development reaches the level of app 100,0\%, comparatively with classic collection methods applied on dead birds, since the parasites leave the dead hosts [2].

The collected material was further studied with the help of lens MBS-9 (ob.14x2) and microscope Novex Holland B (ob. 20-40) WF 10x Din/20mm. 


\section{Results and discussions}

The conducted studies on the diversity of parasitic fauna in pheasants (Chrysolophus amherstiae and Lophura nycthemera) allowed identifying the diverse ectoparasites from: Family Philopteridae - 3 species (Cuclotogaster cinereus, Goniocotes gallinae, Cuclotogaster heterographus); Family Menoponidae - 2 species (Menacanthus stramineus, Menopon gallinae) and Family Ceratophyllidae - one species (Ceratophylus hirundinis) (see Table 1).

There were three species of ectoparasites identified in silver pheasant, all belonging to Family Philopteridae (Cuclotogaster cinereus, Cuclotogaster heterographus Lipeurus caponis) and one species from Family Menoponidae (Eomenacanthus stramineus).

The ordinary pheasant kept in the Zoo from Chişinău city revealed the high diversity of ectoparasites: Family Philopteridae - 2 species (Goniocotes gallinae, Cuclotogaster cinereus); Family Menoponidae - 2 species (Menacanthus stramineus, Menopon gallinae); Family Ceratophyllidae - one species (Ceratophylus hirundinis) and Family Dermanyssidae - 2 species (Dermanyssus gallinae, D. hirundinis).

The study on the ectopasites diversity in quails kept in the Zoo of Chişinău city revealed the high diversity of ectoparasites. Totally, 10 species of ectoparasies have been identified in quails being systematized to the following families: Family Philopteridae - 4 species (Cuclotogaster cinereus, Goniocotes chrysocephalus, Goniodes astrocephalus, Lipeurus caponis); Family Menoponidae - 2 species (Menacanthus abdominalis, Menopon gallinae); Family Ceratophyllidae - 2 species (Ceratophylus gallinae, Ceratophylus hirundinis) and Family Dermanyssidae - 2 species (Dermanyssus gallinae, D. hirundinis).

The studies on identifying the diversity of ectoparasitic zoofauna in ashy partridges allowed tracking 5 species of ectoparasites from the following families: Family Philopteridae - one species (Goniodes dispar); Family Menoponidae - 2 species (Amyrsidea perdicis, Menopon gallinae); Family Ceratophyllidae - one species (Ceratophylus hirundinis) and Family Dermanyssidae - 2 species (Dermanyssus hirundinis).

Table 1. Ectoparasitic fauna in birds of hunting interest from the Zoo of Chişinău city

\begin{tabular}{|c|c|c|c|}
\hline N/o & Host of parasites & $\begin{array}{l}\text { Examined birds } \\
\text { (exemplars) }\end{array}$ & Species of identified parasites \\
\hline & $\begin{array}{l}\text { Lady Amherst's } \\
\text { pheasant }\end{array}$ & 12 & $\begin{array}{l}\text { Cuclotogaster cinereus (Nitzsch, 1866); } \\
\text { Goniocotes gallinae (De Geer, 1778); } \\
\text { Cuclotogaster heterographus (Nitzsch, 1866); } \\
\text { Menacanthus stramineus (Nitzsch,1818); } \\
\text { Menopon gallinae (Linnaeus, 1758); } \\
\text { Ceratophylus hirundinis (Curtis, 1826) }\end{array}$ \\
\hline & Silver pheasant & 16 & $\begin{array}{l}\text { Cuclotogaster cinereus (Nitzsch, 1866); } \\
\text { Goniocotes gallinae (De Geer, 1778); } \\
\text { Cuclotogaster heterographus (Nitzsch, 1866); } \\
\text { Menacanthus stramineus (Nitzsch,1818); } \\
\text { Menopon gallinae (Linnaeus, 1758); } \\
\text { Ceratophylus hirundinis (Curtis, 1826) }\end{array}$ \\
\hline
\end{tabular}




\begin{tabular}{|c|c|c|}
\hline Golden pheasant & 9 & $\begin{array}{l}\text { Cuclotogaster cinereus (Nitzsch, 1866); } \\
\text { Cuclotogaster heterographus (Nitzsch, 1866); } \\
\text { Lipeurus caponis(Linné. 1758); } \\
\text { Eomenacanthus stramineus (Nitzsch, 1818). }\end{array}$ \\
\hline Common pheasant & 18 & $\begin{array}{l}\text { Goniocotes gallinae (De Geer, 1778); } \\
\text { Cuclotogaster cinereus (Nitzsch, 1866); } \\
\text { Menacanthus stramineus (Nitzsch,1818) } \\
\text { Menopon gallinae (Linnaeus, 1758); } \\
\text { Ceratophylus hirundinis (Curtis, 1826); } \\
\text { Dermanyssus gallinae (De Geer, 1778); } \\
\text { D. hirundinis (Duges, 1834). }\end{array}$ \\
\hline Quail & 22 & $\begin{array}{l}\text { Cuclotogaster cinereus (Nitzsch, 1866); } \\
\text { Goniocotes chrysocephalus (Giebel,1874); } \\
\text { Goniodes astrocephalus (Burmeister, 1838); } \\
\text { Lipeurus caponis(Linné. 1758); } \\
\text { Menacanthus abdominalis (Piaget,1880); } \\
\text { Menopon gallinae (Linnaeus, 1758); } \\
\text { Ceratophylus gallinae (Schrank, 1803); } \\
\text { Ceratophylus hirundinis (Curtis, 1826); } \\
\text { Dermanyssus gallinae (De Geer, 1778); } \\
\text { D. hirundinis (Duges, 1834). }\end{array}$ \\
\hline Grey partridge & 8 & $\begin{array}{l}\text { Goniodes dispar (Burmeister, 1838); } \\
\text { Amyrsidea perdicis (Denny,1842); } \\
\text { Menopon gallinae (Linnaeus, 1758); } \\
\text { Ceratophylus hirundinis (Curtis, 1826); } \\
\text { Dermanyssus hirundinis (Duges, 1834) } \\
\end{array}$ \\
\hline Peacock & 7 & $\begin{array}{l}\text { Goniocotes chrysocephalus (Giebel,1874); } \\
\text { Cuclotogaster cinereus (Nitzsch, 1866); } \\
\text { Menopon gallinae (Linnaeus, 1758); } \\
\text { Eomenacanthus stramineus (Nitzsch, 1818); } \\
\text { Amyrsidea perdicis (Denny,1842); } \\
\text { Ceratophylus hirundinis (Curtis, 1826); } \\
\text { Dermanyssus gallinae (De Geer, 1778); } \\
\text { D. hirundinis (Duges, 1834); } \\
\text { Ornithonyssus sylviarum (Canestrini et Fanzago, 1877). }\end{array}$ \\
\hline Chucar partridge & 6 & $\begin{array}{l}\text { Goniocotes chrysocephalus (Giebel,1874); } \\
\text { Goniocotes microthorax (Stephens, 1829); } \\
\text { Cuclotogaster heterographus (Nitzsch, 1866); } \\
\text { Ceratophylus hirundinis (Curtis, 1826); } \\
\text { Menopon gallinae (Linnaeus, 1758); } \\
\text { Menacanthus stramineus (Nitzsch,1818) }\end{array}$ \\
\hline Mandarin duck & 12 & Menopon obscurum, Trinoton querquedulae. \\
\hline Wild goose & 14 & $\begin{array}{l}\text { Anaticola crassicornis, Anatoecus dentatus, Anatoecus } \\
\text { icterodes. }\end{array}$ \\
\hline
\end{tabular}


The study of ectoparasitic fauna diversity in peacocks allowed systematizing to the following families: Family Philopteridae - 2 species (Goniocotes chrysocephalus, Cuclotogaster cinereus); Family Menoponidae - 3 species (Amyrsidea perdicis, Menopon gallinae, Eomenacanthus stramineus); Family Ceratophyllidae - one species (Ceratophylus hirundinis) and Family Dermanyssidae - 3 species (Dermanyssus gallinae, Dermanyssus hirundinis, Ornithonyssus sylviarum).

The study of ectoparasitic fauna diversity in 6 species of partridges kept in captivity allowed systematizing of the identified ectoparasites to the following families: Family Philopteridae - 3 species (Goniocotes chrysocephalus, Goniocotes microthorax, Cuclotogaster heterographus); Family Menoponidae - 2 species (Menopon gallinae, Menacanthus stramineus) and Family Ceratophyllidae - one species (Ceratophylus hirundinis).

The study on diversity of ectoparasites in Mandarin ducks kept in captivity along with other fancy poultry allowed tracking two species of mallophaga from Family Menoponidae (Menopon obscurum, Trinoton querquedulae).

The study on ectoparasitic diversity in wild gooses allowed identifying three species of ectoparasites from Family Menoponidae (Anaticola crassicornis, Anatoecus dentatus, Anatoecus icterodes).

In this way, the parasitological research revealed that the domestic birds as well as the wild ones are infested with multiple parasites and that the majority of identified parasitizes are common for majority of birds of hunting interest as well as for those domestic ones.

The results of the complex parasitological studies in wild birds of hunting interest and in domestic birds revealed that the poliparasitism phenomenon has a permanent character although the poliparasitism structure is in continuous quantitative and qualitative dynamic. The reasons are the continuous contact of wild birds with those domestic ones, irregular disinfestation measures, reducing birds' area for breading and maintenance that ensures the permanent contact with the wild birds as the infestation source.

The studies were carried out within the research project no. 20.80009.7007.12.

\section{Bibliography}

1. Erhan D. Tratat de parazitoze asociate ale animalelor domestice. Chişinău, Î.S. Firma Editorial-Poligrafică „Tipografia Centrală”. 2020, 1040 p.

2. Luncaşu M., Zamornea M. Procedeu de colectare a ectoparaziţilor de la păsări. Brevet de invenţie. $3441 \mathrm{G} 2$, MD, A01 M 1/20 BOPI nr. 12/2007.

3. Olteanu G., Gherman I., Panaitescu D., ș.a. Revista Română de Medicină Veterinară. 1994, Vol. IV. Nr. 3, p. 241-282.

4. Olteanu G., Gherman I., Panaitescu D., ș.a. Revista Română de Parazitologie. 1994, Vol. IV. Nr. 1, p. 4-31.

5. Olteanu G., Panaitescu D., Gherman I. ș.a. Probleme ale parazitozoonozelor spre sfârşit de mileniu în România. Revista Română de Parazitologie. 1995, Vol. V. Nr. 1, p. 1-11.

6. Olteanu G., Panaitescu D., Gherman I. şi colab. Poliparazitismul la om, animale, plante și mediu. Ed. Ceres. București, 2001, 818 p.

7. Olteanu G., Panaitescu D., Gherman I., ș.a. Probleme ale parazitozoonozelor spre sfârşit de mileniu în România. Revista Română de Parazitologie. 1995, Vol. V. Nr. 2, p. 1-19. 
8. Olteanu G., Panaitescu D., Gherman I., ș.a. Unele probleme actuale ale poliparazitismului la om, animale, plante şi mediu în România.Revista Română de Parazitologie. 1993, Vol. III. Nr. 2, p. 3-24.

9. Oprescu I., Cosoroabă I., Dărăbuş G. et. al. Influenţa vitaminelor A, E şi C asupra răspunsului imun faţă de infestaţia cu Dictyocaulus filaria la ovine. Revista Română de Parazitologie. 1999, Vol. IX. Nr. 1, p. 31-33.

10. Rusu Ş., Erhan, D., Savin A., Zamornea M., Rusu V., Railean N., Toderaş I. Parazitofauna, impactul parazitozelor asupra organismului la fazanul comun (Phasianus colchicus L.), profilaxia și tratamentul. Chișinău: S. n., F.E.-P.”Tipografia Centrală”, 2020, 80 p.

11. Şuteu I. Zooparaziţii şi gazdele parazitare. Ed. Genesis. 1998, 400 p.

12. Şuteu I., Cozma V. Parazitologie clinică veterinară. Cluj-Napoca, 2007 a, Vol. I, 316 p.

13. Şuteu I., Cozma V. Parazitologie clinică veterinară. Cluj-Napoca, 2007 b, Vol. II, 349 p.

14. Şuteu I., Cozma V., Gherman C. Probleme actuale privind profilaxia antiparazitară a ecosistemelor. „Ecologia, evoluţia şi ocrotirea diversităţii regnului animal şi vegetal”. Chişinău, 2003, p. $239-246$.

15. Tomșa M. Inspecția și controlul sanitar-veterinar al produselor de origine animală și vegetală. Chișinău, 2016, 648 p.

16. Tomșa M. Siguranța alimentelor. Chișinău, 2018, 620 p.

17. Tomşa M., Bondoc I. Igiena și tehnologia prelucrării produselor şi subproduselor de origine animală. Chișinău, 2014, 472 p.

18. Тодераш И.К. и др. Роль птиц и эктопаразитов в поддержании, возобновлении и возможном появлении новых очагов зоонозных инфекций. Сообщение 1. Buletinul Academiei de Ştiinţe a Moldovei. Ştiinţele vieţii. 2008, nr. 2, p. 4-10.

19. Акбаев М. и др. Паразитология и инвазионные болезни животных. М., 2000, 743 с.

20. Кербабаев Э.Б. и др. Арахноэнтомозы сельскохозяйственных животных. М., 2000, 137 с. 21. Сафиуллин Р. и др. Дракер 10.2 против куриного клеща. Ветеринария. 2013, № 6, с. 32-34. 